\title{
Première contribution à la flore diatomique de l'île Amsterdam (Terres Australes et Antarctiques Françaises)
}

\author{
B. Van de Vijver 1,2 \\ L. Beyens ${ }^{1}$
}

Mots-clés : Diatomées, Ile Amsterdam, eau douce.

Nous présentons la première étude sur les diatomées terrestres et dulçaquicoles de l'Ile Amsterdam située dans l'Océan Indien du Sud. 90 taxons y ont été dénombrés. Cette flore ne présente pas de similitudes avec celle d'autres îles subtropicales telles que l'Ile Maurice ou l'Ile de la Réunion, mais il y a une ressemblance certaine avec la flore diatomique des îles subantarctiques. Une Classifiaction hiérarchique ascendante (CAH) sépare quatre communautés, probablement sur la base de gradients d'humidité et de pH. La communauté à Pinnularia borealis et la communauté à Luticola mutica préfèrent des endroits plutit secs. La communauté à Nitzschia frustulum var. frustulum est retrouvée dans des endroits plus humides, tandis que la communauté à Frustulia rhomboides n'est observée que dans les tourbières du plateau sommital. Cette dernière communauté est caractérisée par la présence d'espèces acidophiles.

\section{First contribution to the diatom flora of the Amsterdam Island (TAAF)}

Keywords : Diatoms, Amsterdam Island, freshwater.

Over 90 taxa have been observed in a first study on the terrestrial and freshwater diatoms of the Amsterdam Island, in the southern Indian Ocean. The observed flora shows little resemblance with that of other subtropical islands (especially Mauritius, Réunion), but there are some similarities with that of subantarctic islands. The cluster analysis revealed 4 assemblages, most problably separated by moisture and $\mathrm{pH}$. The Pinnularia borealis assemblage and the Luticola mutica assemblage prefer dry places. The Nitzschia frustulum var. frustulum assemblages is found on wetter places such as an old block house. The last assemblage is characterized by the presence of many acidophilic taxa and is only reported from the peat area on the central mountain of the island.

\section{Introduction}

L'île Amsterdam (37 47'S-77 34'E) est située à peu près à égale distance de Madagascar, du Continent Antarctique et de 1'Australie dans la région centrale de l'Océan Indien (Fig.1). Cette petite île volcanique présente une longueur de 10 kilomètres, une largeur de

1. Université d'Anvers, Département de Biologie, Unité de Biologie Polaire et Paléobiologie, Groenenborgerlaan 171, B-2020 Anvers, Belgique.

2. Auteur correspondant : University of Antwerp (RUCA), Department of Biology Section Polar Ecology, Limnology and Paleobiology, Groenenborgerlaan 171 B-2020 Antwerp Belgium. E-mail : bartvdv@ruca.ua.ac.be
7 kilomètres et culmine à 881 mètres d'altitude, au Mont de la Dives. La surface totale est de $55 \mathrm{~km}^{2}$. La plus grande partie de la côte est constituée de falaises abruptes.

Du point de vue bioclimatique, l'île bénéficie d'un climat doux avec des étés austraux plutôt secs et chauds et avec des hivers plutôt tempérés et humides. La température moyenne est $13,8 \mathrm{C}$ avec un minimum de 11,2 $\mathrm{C}$ en août et un maximum de $17 \mathrm{C}$ en février. Les précipitations annuelles sont de l'ordre de $1115 \mathrm{~mm}$ et montrent un minimum en été (février-mars). Selon Stonehouse (1982), l'île Amsterdam appartient à la zone chaude tempérée de la province subantarctique. Elle fait partie des Terres Australes et Antarctiques Françaises (TAAF). 


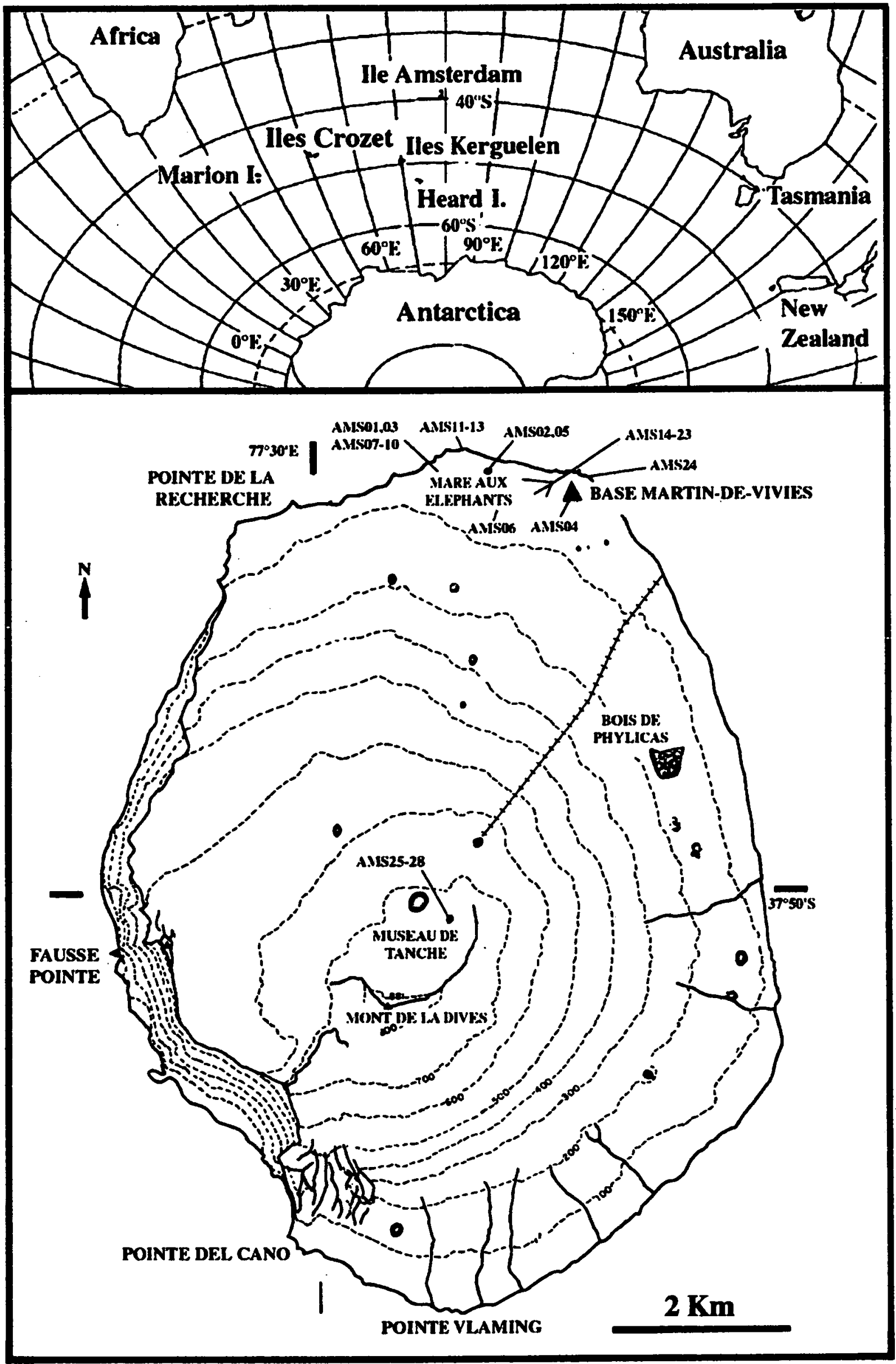

Fig. 1. Carte schématique de la localisation des sites d'échantillonnages (d'après Frenot \& Valleix 1990).

Fig. 1. Sketch map of the study site with the location of the different sampling points (after Frenot \& Valleix 1990). 
Le réseau hydrographique est très peu développé (Frenot \& Valleix 1990). Les fortes pluies forment de temps en temps des petits torrents violents qui dévalent les pentes et rejoignent alors seulement la côte. Ces torrents présentent un caractère semi-permanent, uniquement à l'Ouest et au Sud-Ouest de l'île, où ils sont alimentés par les tourbières du plateau sommital.

La végétation de l'île est constituée d'espèces propres à la région subantarctique mais contient également de nombreuses espèces introduites (Jolinon 1987). Ainsi, les communautés végétales présentes autour de la base sont composées essentiellement de plantes allochtones résistant bien au pâturage et au piétinement par les bovins, eux aussi introduits : Holcus lanatus, Leontodon taraxoides et Cirsium vulgare. Les rochers sont couverts avec de petites mousses (e.g. Bryum isleanum Besch., Raphidorrhynchium aurescens (Jaeg.) Blesch). Tixier (1980) a publié une liste de 20 espèces de mousses.

A notre connaissance, aucune étude sur les diatomées n'avait été entreprise jusqu'à présent sur l'île Amsterdam. En 1971, Pierre \& Noel publiaient une étude sur les formations dulçaquicoles de l'île, men- tionnant les algues rencontrées mais sans aborder les diatomées. Jusqu'à maintenant, c'est le seul travail qui existe sur des recherches hydrobiologiques sur l'île.

Une courte visite de l'île le 12 février 1998 nous a permis d'effectuer quelques prélèvements dans les environs de la Base Martin-de-Viviès en vue d'une étude préliminaire de la flore diatomique terrestre et dulçaquicole. Nous avons pu également disposer dans cette étude de 4 échantillons anciens, récoltés en 1969 dans le cratère du Museau de Tanche, sur le plateau sommital.

\section{Matériel et méthodes}

Vingt-quatre échantillons ont été prélevés aux alentours de la base (Fig.1). Selon la carte des sols réalisée par Frenot \& Valleix (1990), la région est composée de sols peu organiques, de faible épaisseur et fortement érodés, suite à l'impact de nombreux incendies et, plus récemment, de bovins (Tréhen et al. 1990). Tous les grandes types d'habitats de ce secteur de l'île ont été échantillonnés. La plupart des échantillons proviennent de mousses sèches, réhumectées de temps en temps par de fortes pluies. Le tableau 1 résume les principales caractéristiques des prélèvements.

Tableau 1. Liste des prélèvements analyés.

Table 1. List of all analyzed samples.

\begin{tabular}{ll}
\hline Les prélèvements aux alentours de la Base \\
AMS01 & rochers avec des mousses \\
AMS02 & Mare aux Eléphants, $\mathrm{pH}=7,2$, conductivité = 1870 $\mu \mathrm{S}$ \\
AMS03 & lithotelme \\
AMS04 & bassin d'eau (en béton) dans la base, $\mathrm{pH}=8$, conductivité = 160 \\
AMS05 & abreuvoir métallique pour les vaches \\
AMS06 & petit trou de la terre dedans, pas de végétation \\
AMS07 & lithotelme, mousses mouillé par la pluie \\
AMS08 & lithotelme sans mousses \\
AMS09 & rochers avec des mousses \\
AMS10 & rochers avec des mousses \\
AMS11 & falaise à 50 m de la mer \\
AMS12 & falaise à 50 m de la mer, présence des lichens \\
AMS13 & rochers avec des mousses \\
AMS14 & abri en béton, petite cave, hépatique dans petit rayon de lumière \\
AMS15 & abri en béton, même hépatique, mais en pleine lumière \\
AMS16 & abri en béton, petite falaise avec des mousses \\
AMS17 & abri en béton, petite falaise couverte avec des algues vertes \\
AMS18 & rochers avec des mousses \\
AMS19 & rochers avec des mousses \\
AMS20 & lithotelme, avec de l'herbe \\
AMS21 & mousses \\
AMS22 & mousses humides \\
AMS23 & mousses humides \\
AMS24 & algues vertes et cyanobactéries sur terre nue \\
Les prélèvements sur le plateau sommital \\
AMS25 & tourbe, Museau de Tanche \\
AMS26 & tourbe, Museau de Tanche \\
AMS27 & tourbe, Museau de Tanche \\
AMS28 & tourbe, Museau de Tanche
\end{tabular}


Les échantillons ont été oxydés par $\mathrm{H}_{2} \mathrm{O}_{2}$ et $\mathrm{KMnO}_{4}$ (Van der Werff 1955). Les diatomées traitées ont ensuite été montées dans du Naphrax ${ }^{\circ}$. Au total, 500 valves ont été dénombrées et identifiées sur chaque lame (microscope Leitz Orthoplan équipé avec une optique à contraste de phase). Nous avons ainsi pu estimer les fréquences relatives des principaux taxons. Les taxons plus rares ont été observés en balayant systématiquement toutes les lames au fort grossissement. La nomenclature adoptée repose sur les travaux de Krammer \& Lange-Bertalot (1986-1991) et de LangeBertalot (1993) sauf pour les genres Luticola et Diadesmis (Round et al. 1990). Pour le genre Pinnularia, la nomenclature de Krammer (1992) a été employée et pour le genre Achnanthes celle de Lange-Bertalot \& Krammer (1989). Pour faciliter les comparaisons avec la littérature ancienne sur les autres îles subantarctiques françaises (Crozet, Kerguelen), nous employons ici les noms anciens de Fragilaria vaucheriae (Kütz.) Petersen et Fragilaria vaucheriae var. longissima Manguin au lieu de Fragilaria capucina var. vaucheriae (Kütz.) Lange-Bertalot.

Une classification hiérarchique ascendante (CAH), basée sur le carré de la distance euclidienne, a été réalisée afin d'ordonner les échantillons de manière objective en fonction de la structure de leurs communautés diatomiques (Kovach Computing Services 1993). Les communautés sont nommées d'après leurs taxons principaux. La distribution des diatomées dans les différentes communautés de l'île Amsterdam a été analysée au moyen d'une Analyse en Composantes Principales (ACP) sur les données transformées en logarithme normal (CANOCO vs. 3.1, ter Braak 1987).

\section{Résultats}

\subsection{Composition floristique}

Dans cette première étude sur les diatomées de l'île Amsterdam, nous avons dénombré 90 taxons (68 espèces, 20 variétés et 2 formes), dont 6 pouvant être considérés comme des contaminants marins (Tableau 2). Ils se répartissent parmi 22 genres. Les genres $\mathrm{Na}$ vicula (20 taxons), Pinnularia (13 taxons), Achnanthes (11 taxons) et Nitzschia (10 taxons) sont les mieux représentés. L'analyse quantitative des diatomées (Fig.2) montre que Pinnularia est le genre le plus couramment observé puisqu'il représente $27,2 \%$ des valves dénombrées, suivi par Luticola $(16,9 \%)$ et Achnanthes $(14,6 \%)$. Cette dominance de Pinnularia contraste avec la situation sur les îles subantarctiques où $A c h$ nanthes est le plus abondant (Van de Vijver \& Beyens 1996, Van de Vijver et al. 1998). Cette situation peut s'expliquer par l'absence à Amsterdam de ruisseaux

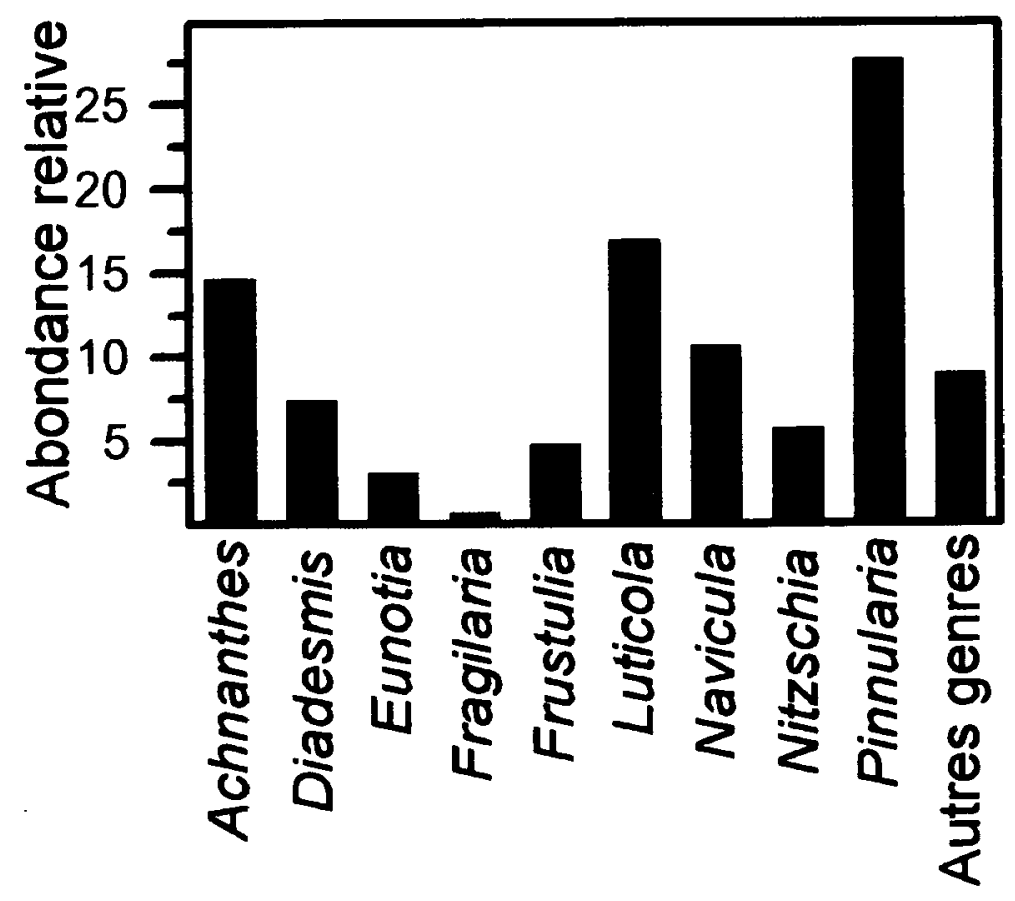

Fig. 2. Importance relative des principaux genres de diatomées observés dans cette étude.

Fig. 2. Relative importance of the main diatom genera observed in this study.

oligotrophes qui constituent en milieu subantarctique, un environnement favorable pour des taxons du genre Achnanthes.

En concordance avec Round et al. (1990), on propose de transférer Navicula arcuata Heiden du genre $\mathrm{Na}$ vicula au genre Diadesmis. La nouvelle combinaison est alors :

\section{Diadesmis arcuata (Heiden) Van de Vijver \& Beyens nov. comb.}

Il y a des auteurs qui sont d'opinion que $D$. arcuata est simplement une forme de D. contenta (Grun.) Mann. Il y a quand même des différences comme la taille plus robuste qui justifient l'espèce $D$. arcuata. On propose alors de garder $D$. arcuata comme espèce et pas comme forme de $D$. contenta.

La plupart des taxons que nous avons observés sont considérés dans la littérature comme des espèces cosmopolites. Deux taxons du genre Achnanthes appartiennent à des milieux insulaires vrais : Achnanthes pericava Carter à Tristan da Cunha (Carter 1966) et Achnanthes islandica Oestrup en Islande (Oestrup 1918). Pinnularia microstauron var. elongata Manguin, Achnanthes subatomoides (Hust.) Lange-Bertalot, Diatomella hustedtii Manguin et Diadesmis arcuata (Heiden) Van de Vijver \& Beyens sont des taxons (sub)-antarctiques, qu'on trouve en abondance sur les Iles Kerguelen (Le Cohu \& Maillard 1986) et Crozet 
Tableau 2. Liste des taxons observées dans cette étude.

Table 2. List of all diatom taxa observed in this study.

Achnanthes biasolettiana Grunow

Achnanthes coarctata (Brébisson) Grunow

Achnanthes delicatula (Kützing) Grunow var. delicatula

Achnanthes islandica Oestrup

Achnanthes lanceolata (Brébisson) Grunow var. lanceolata

Achnanthes minutissima Kützing var. minutissima

Achnanthes ninkei Guermeer \& Manguin

Achnanthes oblongella Oestrup

Achnanthes pericava Carter

Achnanthes subatomoides (Hustedt) Lange-Bertalot

Achnanthes A

Amphora veneta Kützing

Brachysira minor (Krasske) Lange-Bertalot

Caloneis bacillum (Grunow) Cleve

Campyloneis grevillei (W. Smith) Grunow

Cocconeis costata Gregory

Cocconeis neodiminuta Krammer

Cocconeis scutellum Ehrenberg

Diadesmis arcuata (Heiden) Van de Vijver \& Beyens

Diadesmis contenta (Grunow) Mann

Diadesmis contenta f. undulata (Manguin) Van de Vijver \& Beyens

Diatomella hustedtii Manguin

Eunotia bilunaris (Ehrenberg) Mills var. bilunaris

Eunotia fallax Cleve-Euler

Eunotia muscicola Krasske var. muscicola

Eunotia nymanniana Grunow

Eunotia paludosa Grunow var. paludosa

Fragilaria construens f. venter (Ehrenberg) Hustedt

Fragilaria fasciculata (Agardh) Lange-Bertalot

Fragilaria pinnata Ehrenberg var. pinnata

Fragilaria ulna (Nitzsch) Lange-Bertalot var. ulna

Fragilaria vaucheriae (Kützing) Petersen

Fragilaria vaucheriae var. longissima Manguin

Frustulia homboides (Ehrenberg) De Toni var. homboides

Gomphonema parvulum (Kützing) Kützing var. parvulum

Gomphonemopsis littoralis (Hendey) Medlin

Gomphonemopsis pseudoexigua

Hantzschia amphioxys (Ehrenberg) W. Smith

Luticola goeppertiana (Bleisch) Mann

Luticola mutica (Kützing) Mann var. mutica

Luticola nivalis (Ehrenberg) Mann

Luticola suecorum var. dismutica (Hustedt) Mann

Luticola ventricosa (Kützing) Mann

Melosira A

Navicula agrestis Hustedt
Navicula atomus (Kützing) Grunow var. atomus

Navicula atomus var. permitis (Hustedt) Lange-Bertalot

Navicula bryophila Petersen

Navicula cryptocephala Kützing

Navicula fossalis Krasske var. fossalis

Navicula genustriata Hustedt

Navicula gregaria Donkin

Navicula minima Grunow

Navicula perminuta Grunow

Navicula saprophila Lange-Bertalot

Navicula seminulum Grunow

Navicula soehrensis Krasske var. soehrensis

Navicula subtillissima Cleve

Navicula tenelloides Hustedt

Navicula veneta Kützing

Navicula A

Navicula B

Nitzschia communis Rabenhorst

Nitzschia debilis Arnott

Nitzschia frustulum (Kützing) Grunow var. frustulum

Nitzschia inconspicua Grunow

Nitzschia palea (Kützing) W. Smith

Nitzschia perspicua Cholnoky

Nitzschia pusilla Grunow

Nitzschia tubicola Grunow

Nitzschia A

Nitzschia B

Orthoseira roeseana (Rabenhorst) O'Meara

Pinnularia borealis var. scalaris (Ehrenberg) Rabenhorst Pinnularia divergentissima var. minor (Grunow) Cleve

Pinnularia intermedia (Lagerstedt) Cleve

Pinnularia kolbei Manguin

Pinnularia microstauron (Ehrenberg) W. Smith

Pinnularia obscura Krasske

Pinnularia rupestris Hantzsch

Pinnularia similiformis Krammer

Pinnularia sinistra Krammer

Pinnularia subcapitata Krammer

Pinnularia submicrostauron Schroeter

Pinnularia termitina (Ehrenberg) Patrick

Rhopalodia rupestris (W. Smith) Krammer

Stauroneis anceps Ehrenberg

Stauroneis kriegeri Patrick

Stauroneis producta Grunow

Thalassiosira A
(Pierre 1977, Van de Vijver \& Beyens soumis). Au contraire, des similitudes avec des îles subtropicales relativement proches telles que l'Ile de la Réunion (Van de Vijver, résultats non-publiés) ou l'Tle Maurice (Coste \& Ricard 1982a, 1982b) n'apparaissent en aucune manière.
Parmi les taxons les plus abondamment observés se trouvent surtout des espèces aérophiles comme Pinnularia borealis var. scalaris (Ehrenb.) Rabenhorst, Luticola mutica (Kütz.) Mann var. mutica, Achnanthes coarctata (Bréb.) Grunow, Diadesmis contenta (Grun.) Mann et Hantzschia amphioxys (Ehrenb.) W. Smith. 


\subsection{Analyse des communautés}

Les résultats de la $\mathrm{CAH}$ révèlent deux groupes d'échantillons (Fig. 3). Un premier groupe rassemble les 24 échantillons prélevés en 1998 qui se répartissent en trois communautés : A 1.1 : la communauté à Nitzschia frustulum var. frustulum, A2.1 : la communauté à Pinnularia borealis; A2.2 : la communauté à Luticola mutica. Le deuxième groupe ne contient que les 4 échantillons prélevés en 1969 et constitue à lui seul la communauté à Frustulia rhomboides var. rhomboides (B).

La figure 4 montre l'abondance relative des taxons dominants et subdominants dans les différentes communautés.

La communauté à Pinnularia borealis est représenté par 14 échantillons. Pinnularia borealis est bien sûr le taxon dominant de cette communauté. Mais d'autres

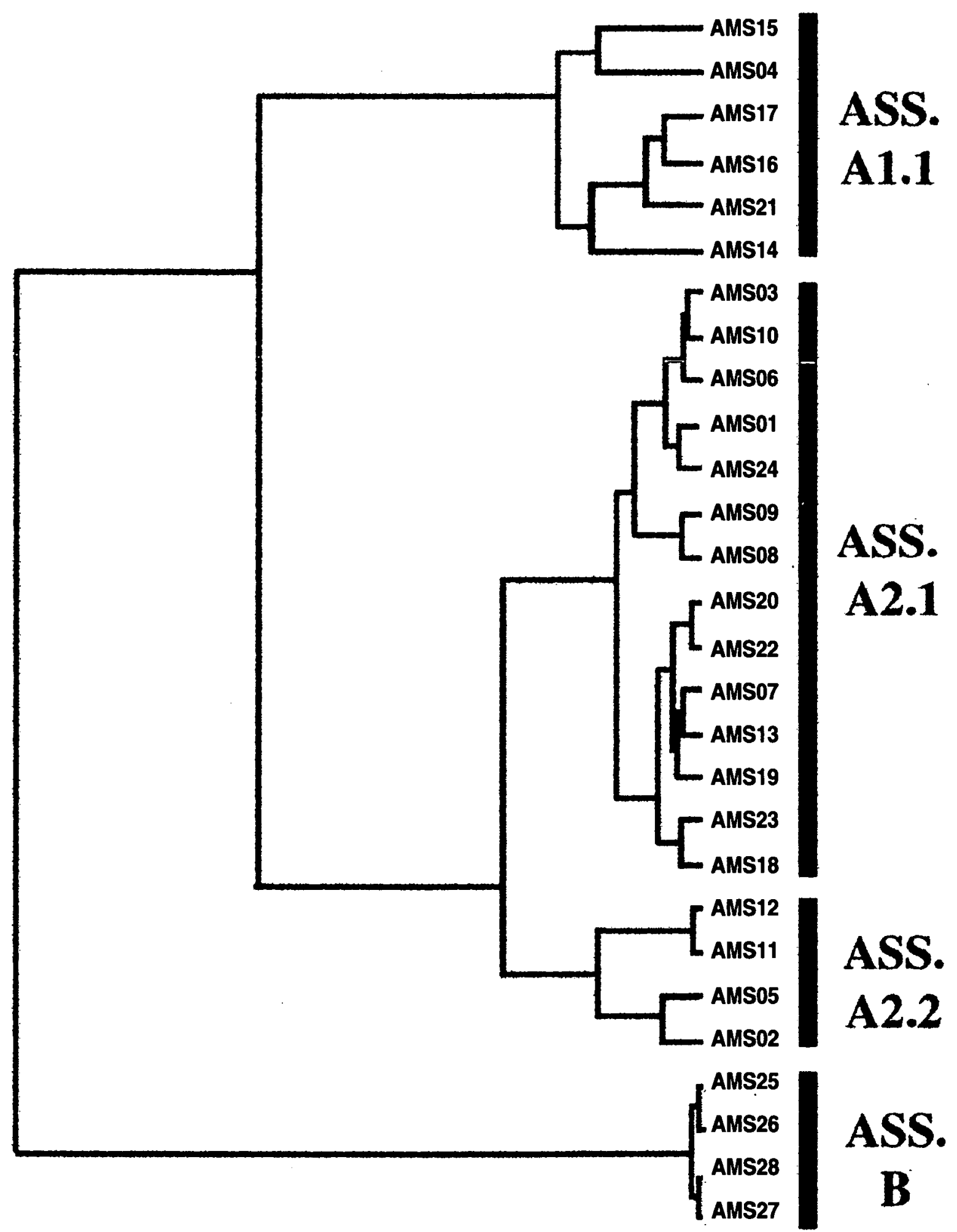

Fig. 3. CAH, basée sur la composition des taxons. Les communautés obtenues sont marquées à droite.

Fig. 3. Cluster analysis based on species composition. The different clusters are marked on the right side. 


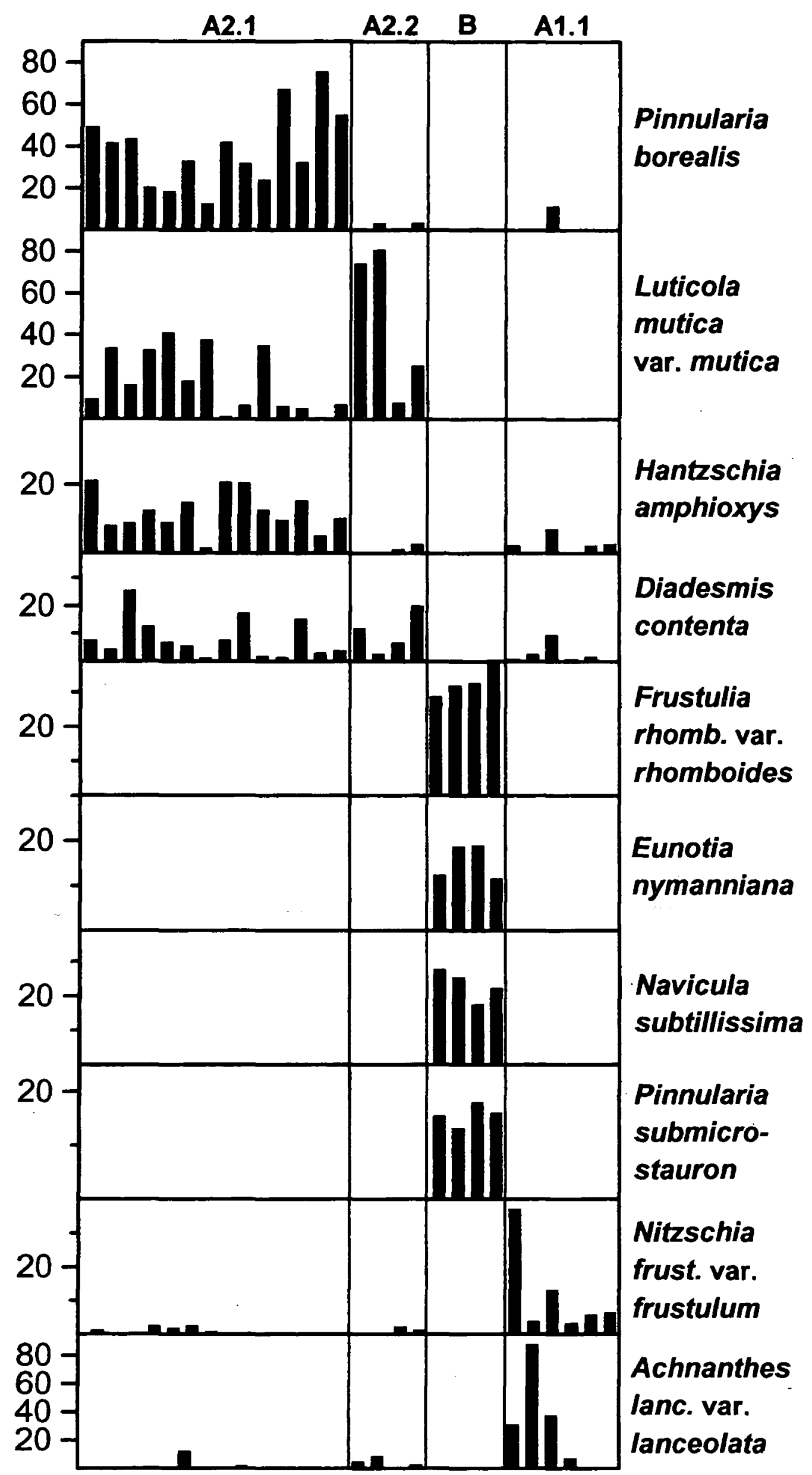

Fig. 4. Abondance relative moyenne des principaux taxons dans les différentes communautés. Les taxons sont classés suivant leur présence dans les différents assemblages en partant de celui à Pinnularia borealis.

Fig. 4. Mean relative abundance of the most important taxa in each cluster. Taxa are grouped according to their occurrence in the different assemblages starting with the Pinnularia borealis assemblage. 
taxons tels que Luticola mutica var. mutica, Hantzschia amphioxys et Diadesmis contenta sont également bien représentés.

La communauté à Luticola mutica var. mutica ne contient que 4 échantillons, dominés par Luticola mutica var. mutica avec des abondances relatives qui atteignent parfois $80 \%$ (AMS11: 80,4\%). Les taxons subdominants sont Achnanthes islandica, Diadesmis contenta et Nitzschia palea (Kütz.) W. Smith. Malgré une forte présence de Luticola mutica dans la communauté à $P$. borealis, il faut néanmoins séparer la communauté à $L$. mutica en raison de l'absence presque totale de $P$. borealis et Hantzschia amphioxys.

Les deux autres communautés ont une composition complètement différente. Les quatre échantillons de la communauté à Frustulia rhomboides var. rhomboides proviennent du plateau sommital de l'île, i.e. le $\mathbf{M u}$ seau du Tanche. Ils sont caractérisés par une dominance de $F$. rhomboides (Ehre.) De Toni var. rhomboides, Eunotia nymanniana Grunow, Navicula subtillissima Cleve et Pinnularia submicrostauron Schroeter.

Dans la communauté à Nitzschia frustulum var. frustulum, les taxons principaux sont Nitzschia frustulum (Kütz.) Grunow var. frustulum et Achnanthes lanceolata (Bréb.) Grunow var. lanceolata. Dans cette communauté se trouve également une espèce de Melosira qui est probablement nouvelle pour la science. La description de cette espèce sera publiée ultérieurement.

L'analyse en composantes principales (PCA) confirme bien la ségrégation de ces différentes communautés (Fig. 5). Les deux axes expliquent environ $49,2 \%$ de l'inertie totale. Les deux communautés qui montrent une flore diatomique aérophile sont positionnées à gauche et au milieu du premier plan factoriel. Les échantillons les plus riches en Pinnularia borealis, Hantzschia amphioxys et Luticola mutica var. mutica présentent des scores fortement négatifs sur le premier axe. Compte tenu de ce que l'on sait de l'écologie de ces espèces, il est vraisemblable que ce premier axe indique un gradient d'humidité.

La communauté à Frustulia rhomboides var. rhomboides est bien séparée des trois autres et montre une composition floristique typique. Elle n'est retrouvée que dans la tourbière sur le plateau culminant de l'île. Les quatre échantillons viennent du lac cratère du $\mathrm{Mu}$ seau de Tanche. Ce petit lac est entouré par des sols tourbeux, couverts de mousses et de Sphaignes. Pierre $\&$ Noel (1971) donnent un valeur du pH entre 4.5 et 5 . Cela peut indiquer que l'axe vertical reflète probablement un gradient de $\mathrm{pH}$.
La dernière communauté (i.e. à Nitzschia frustulum var. frustulum) est aussi bien séparée des autres. Cette communauté groupe tous les échantillons pris dans un milieu non-acide mais bien humide. Cinq échantillons viennent du même endroit, c'est-à-dire un abri en béton environné d'une végétation de Cirsium. Le sixième échantillon appartenant à cette communauté a été prélevé dans un bassin d'eau sur la base.

\section{Discussion}

Ces communautés diatomiques s'organisent selon un gradient allant de la côte vers le plateau tourbeux sommital.

La zone côtière d'Amsterdam est composée de falaises et de rares petites plages rocheuses. Les falaises autour l'île sont couvertes de lichens et de mousses, exposés aux vents et aux embruns. Ceci crée des circonstances assez sèches mais aussi assez riches en ions, favorisant les taxons de la communauté à Luticola mutica var. mutica, comme Achnanthes islandica qui était typique pour la zone côtière en Islande (Oestrup 1918). La «Mare aux éléphants» est un petit étang, peu profond situé en arrière d'une petite plage rocheuse, à proximité de la mer. Un grand nombre d'otaries (Arctocephalus tropicalis) fréquentent cette plage et se baignent dans ce petit étang. Pierre \& Noel (1971) avaient déjà constaté que ces animaux apportent une grande quantité de matières organiques. Ils contribuent également à l'enrichissement en chlorures puisque les mesures de Pierre \& Noel (1971) montraient une résistivité de l'ordre de $1250 \Omega / \mathrm{cm}$ et une chlorinité d'environ $175 \mathrm{mg} / \mathrm{l}$ d'ions chlore. Ceci est confirmé par l'abondance relative considérable de Nitzschia palea (Kütz.) W. Smith $(51,8 \%$ ) et Navicula saprophila Lange-Bertalot $(12 \%)$. Ces deux taxons sont considérés par de nombreux auteurs comme polysaprobiques, tolérants des degrés élevés de pollution et des fortes variations osmotiques (Krammer \& Lange-Bertalot 1986-1991, Van Dam et al. 1994). Malheureusement, notre séjour sur l'Ile Amsterdam a été de trop courte durée pour nous permettre d'effectuer quelques analyses physico-chimiques plus élaborées. Cela nous aurait permis de confirmer cette hypothèse.

Dès que l'on s'éloigne un peu de la côte, la fréquence de Luticola mutica var. mutica et Achnanthes islandica diminue très nettement tandis que la présence de Hantzschia amphioxys et Pinnularia borealis var. scalaris augmente. La communauté à Luticola mutica est donc progressivement remplacée par la communauté à Pinnularia borealis. Les échantillons de cette communauté ont été prélevés à partir des mousses sèches, poussant sur des rochers et dans des lithotelmes 


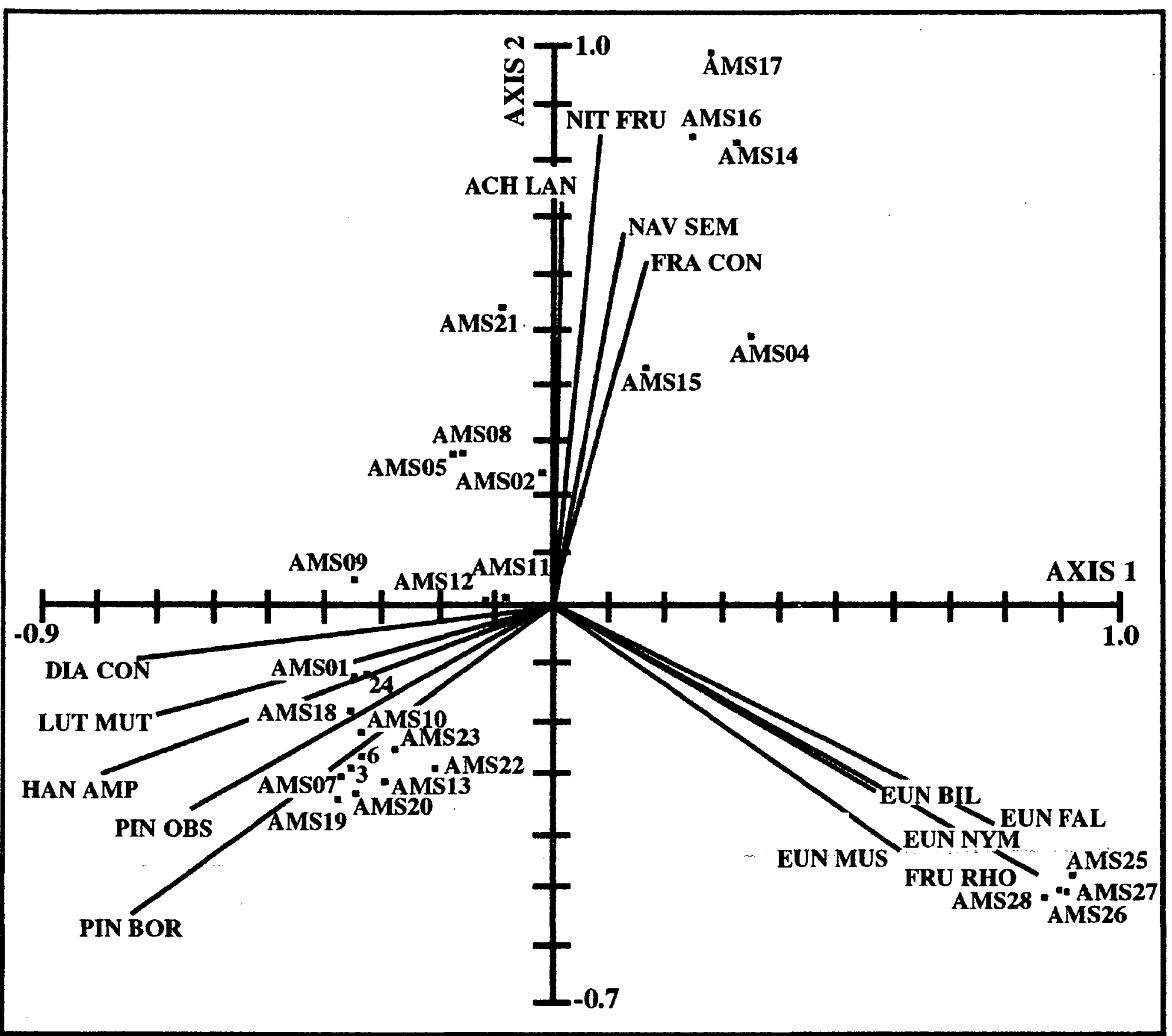

Fig. 5. Premier axe factoriel de l'ACP, montrant les principaux taxons : (ACHLAN = Achnanthes lanceolata var. lanceolata, DIACON = Diadesmis contenta, EUNBIL = Eunotia bilunaris var. bilunaris, EUNMUS = Eunotia muscicola var. muscicola, EUN$\mathrm{NYM}=$ Eunotia nymanianna, $\mathrm{EUNPAL}=$ Eunotia paludosa var. paludosa, $\mathrm{FRACON}=$ Fragilaria construens $\mathrm{f}$. venter, $\mathrm{FRURHO}$ $=$ Frustulia rhomboides var. rhomboides, HANAMP = Hantzschia amphioxys, LUTMUT = Luticola mutica var. mutica, NAVSEM = Navicula seminulum, NITFRU = Nitzschia frustulum var. frustulum, PINBOR = Pinnularia borealis var. scalaris, $\mathrm{PINOBS}=$ Pinnularia obscura).

Fig. 5. PCA analysis plotting the first axis (horizontal) against the second (vertical. The main taxa are represented (ACHLAN $=$ Achnanthes lanceolata var. lanceolata, DIACON = Diadesmis contenta, EUNBIL $=$ Eunotia bilunaris var. bilunaris, EUNMUS $=$ Eunotia muscicola var. muscicola, EUNNYM = Eunotia nymanianna, EUNPAL = Eunotia paludosa var. paludosa, $\mathrm{FRACON}=$ Fragilaria construens $\mathrm{f}$. venter, FRURHO = Frustulia rhomboides var. rhomboides, HANAMP = Hantzschia amphioxys, LUTMUT = Luticola mutica var. mutica, NAVSEM = Navicula seminulum, NITFRU = Nitzschia frustulum var. frustulum, PINBOR = Pinnularia borealis var. scalaris, PINOBS = Pinnularia obscura) 
(flaques d'eau temporaires dans des rochers approfondis, alimentées par des pluies). Tous les lieux d'échantillonage restent très secs sauf pendant des périodes de fortes pluies. Ce type de végétation couvre une grande partie de l'Ile Amsterdam à basse altitude.

Des changements dans l'humidité et le $\mathrm{pH}$ sont responsables pour la présence des communautés exceptionnelles. Les conditions stationnelles, notamment d'humidité et de $\mathrm{pH}$, jouent un rôle important dans la structure des communautés diatomiques. Ainsi, sur le plateau tourbeux situé en altitude, les.conditions acides et humides favorisent l'existence de la communauté à Frustulia rhomboides var. rhomboides. Les autres espèces participant à cette communauté, Navicula subtillissima, Pinnularia submicrostauron et Eunotia nymanniana, sont toutes connues pour préférer des conditions acides (Krammer 1992, Krammer \& LangeBertalot 1986-1991). C'est une situation que l'on retrouve souvent dans les tourbières d'altitude, mais une deuxième campagne de récolte sur le plateau sommital de l'île Amsterdam sera nécessaire pour mieux caractériser la flore diatomique de ses milieux d'altitude.

Les mousses constamment humides et les petits bassins d'eau sur la base représentent également un milieu spécial sur cette île où l'eau est un facteur limitant important. La communauté à Nitzschia frustulum var. frustulum n'a été observée que dans ce type de situation. La présence dans cette communauté de taxons tels que Nitzschia frustulum var. frustulum et Achnanthes lanceolata var. lanceolata, connus des endroits aérophiles mais bien humides (Van Dam et al. 1994), confirment cette hypothèse.

\section{Conclusion}

Une flore diatomique bien diversifiée a été observée dans les 28 échantillons. La flore comprend des éléments des îles bien isolées comme Tristan da Cunha et des îles subantarctiques, notamment des Iles Kerguelen et de Crozet. Les analyses des communautés montrent une division des échantillons en 4 communautés. Ces communautés représentent une zonation floristique de l'île, déterminée par l'humidité et le $\mathrm{pH}$.

\section{Remerciements}

Cette étude a été réalisée avec le support logistique et financier de 1'Institut Français pour la Recherche et la Technologie Polaire (IFRTP), dans le cadre du programme d'écologie terrestre $\mathrm{n}^{\circ} 136$ (Y. Frenot). On remercie Y. Frenot pour la correction du texte français. Un financement complémentaire a été fourni par la Fondation des Recherches Scientifiques, Flandre (FWO). Dr. J.-F. Pierre nous a très gentiment donné les échantillons de 1969. Bart Van de Vijver est aspirant-chercheur du FWO, Flandre.

\section{Travaux cités}

Carter J.R. 1966. - Some freshwater diatoms of Tristan da Cunha and Gough Islands. Nov. Hedw., $11: 443-481$.

Coste M. \& Ricard M. 1982a. - A systematic approach to the freshwater diatoms of Seychelles and Mauritius Islands. 7 th Diat. Symp. : 307-326.

Coste M. \& Ricard M. 1982b. - Contribution à l'étude des diatomées d'eau douce des Seychelles et de l'Ile Maurice. Crypt. Algol., 3 (4) :279-313.

Frenot Y. \& Valleix T. 1990. - Carte des sols de l'nle Amsterdam (TAAF). C.N.F.R.A., $59: 49$ p.

Jolinon J.C. 1987. - Les Phanérogames adventices sur l'Ile Amsterdam. C.N.F.R.A., $58: 183-188$.

Kovach Computing Services 1993. - Multivariate statistical package version 2.1 users' manual.

Krammer K. 1992. - Pinnularia. Eine Monographie der Europäischen Taxa. Bibliotheca Diatomologica, 26 : 1-353.

Krammer K. \& Lange-Bertalot H. 1986-1991. - Süsswasserflora von Mitteleuropa Bacillariophyceae Teil 1-4. G. Fisher Verlag, Stuttgart : 2486 p.

Lange-Bertalot H. 1993. - 85 neue Taxa und über 100 weitere neu definierte Taxa ergänzend zür Süsswasserflora von Mitteleuropa Vol 2/1-4. Bibliotheca Diatomologica, 27 : 453 p.

Lange-Bertalot H. \& Krammer K. 1989. - Achnanthes, eine Monographie der Gattung. Bibliotheca Diatomologica, 18 : 1-393.

Le Cohu R. \& Maillard R. 1986. - Diatomées d'eau douce des îles Kerguelen à l'exclusion des Monoraphidées. Annls. Limnol., $22(2): 99-118$.

Oestrup E. 1918. - Freshwater diatoms from Iceland. In : Rosenvings \& Warming, Botany of Iceland 2/1 (5) : 1-96 avec 5 planches.

Pierre J.-F. \& Noel P. 1971. - Recherches hydrobiologiques à l'île Amsterdam : campagne 1969-1970. Bull. Ac. Soc. Lorr. Sci., $10(2): 101-111$.

Pierre J.-F. 1977. — Les algues des eaux courantes de l'Ile de la Possession, Archipel Crozet. Bull. Soc. Phycol. Fr., 22 : 79-86.

Round F.E., Crawford R.M. \& Mann D.G. 1990. - The diatoms, biology and morphology of the genera. Cambridge University Press, Cambridge.

Stonehouse B. 1982. - La zonation écologique sous les hautes latitudes australes. C.N.F.R.A., $51: 532-537$.

ter Braak C.F.T. 1987. - Canoco - a Fortran program for canonical community ordination by (partial) (detrended) (canonical) correspondance analysis, principal components analysis and redundancy analysis. Wageningen ITI-TNO.

Tixier P. 1980. - Bryophyta exotica. VI Données muscologiques nouvelles sur l'île Amsterdam (Océan Indien du Sud). Nov. Hedw., 32 : 483-491.

Tréhen P., Frenot Y., Lebouvier, M. \& Vernon P. 1990. — Invertebrate fauna and their role in the degradation of cattle dung at Amsterdam Island. In : Kerry K.R. \& Hempel G. (eds.), Antarctic Ecosystems. Ecological Change and Conservation. Springer-Verlag, Berlin : 337-346.

Van Dam H., Mertens A. \& Sinkeldam J. 1994. - A coded checklist and ecological indicator values of freshwater diatoms from the Netherlands. Neth. J. Aq. Ecol., 28 : 117-133.

Van der Werff A. 1955. - A new method of concentrating and cleaning diatoms and other organisms. Verh. Int. Verein. Theor. Angew. Limnol., 12 : 276-277.

Van de Vijver B. \& Beyens L. 1996. - Freshwater diatom communities of the Strømness Bay area, South Georgia. Ant. Sci., 8 (4) : 359-368.

Van de Vijver B. \& Beyens L. (soumis) - Moss diatom communities of lle de la Possession (Crozet, Subantarctica). Polar Biol.

Van de Vijver B, Beyens L., Gloaguen J.C. \& Frenot Y. 1998. — La flore diatomique de quelques sédiments tourbeux holocènes des îles Kerguelen. Annls. Limnol., 34 (1) : 3-11. 